\title{
Symmetry-induced tunneling in one-dimensional disordered potentials
}

\author{
E. Diez, ${ }^{1}$ F. Izrailev, ${ }^{2}$ A. Krokhin,${ }^{3}$ and A. Rodriguez ${ }^{1,4}$ \\ ${ }^{1}$ Departamento de Física Fundamental, Universidad de Salamanca, Salamanca E-37008, Spain \\ ${ }^{2}$ Instituto de Física, Universidad Autónoma de Puebla, Apartado Postal J-48, Puebla 72570, Mexico \\ ${ }^{3}$ Department of Physics, University of North Texas, P.O. Box 311427, Denton, Texas 76203, USA \\ ${ }^{4}$ Department of Physics and Centre for Scientific Computing, University of Warwick, Coventry CV4 7AL, United Kingdom
}

(Received 8 April 2008; revised manuscript received 27 May 2008; published 17 July 2008)

\begin{abstract}
A nontraditional mechanism of tunneling at macroscopic distances is proposed for a wave packet localized in a one-dimensional disordered potential with mirror symmetry, $V(-x)=V(x)$. Unlike quantum tunneling through a regular potential barrier, which occurs only at the energies lower than the barrier height, the proposed mechanism of tunneling exists even for weak white-noise-like scattering potentials. It also exists in classical circuits of resonant contours with random resonant frequencies. The latter property may be used as a new method of secure communication, which does not require coding and decoding of the transmitting signal.
\end{abstract}

DOI: 10.1103/PhysRevB.78.035118

PACS number(s): 72.15.Rn, 72.10.Bg, 72.80.Ng

\section{INTRODUCTION}

It is well known that all quantum states in a onedimensional white-noise potential are strongly localized and quantum transport is limited by the localization length $l(E)$. Statistical correlations in the disordered potential may give rise to a discrete $\operatorname{set}^{1}$ or to a continuum of delocalized states $^{2,3}$ for short- or long-range correlation, respectively. Correlations are a manifestation of the local properties of a random potential. The symmetry is a global property; therefore, its effect on the transport may be even stronger.

Here we propose a symmetry-driven mechanism of tunneling, which is specific for the random potentials only. Usually, the symmetry is considered to be an irrelevant property since a localized state is formed due to some local fluctuations and it is insensitive to global behavior of the potential outside the radius of localization. However, the symmetry of the potential, $V(-x)=V(x)$, leads to a definite parity of the wave functions. Even or odd parity means that the wave function has two equal peaks with half-width $\sim l(E)$ centered at the symmetric points. A symmetry-induced correlation between these peaks gives rise to the mechanism of tunneling of a wave packet (or excitation), independently of how far apart the peaks are. Due to this mechanism, a wave packet injected at some point tunnels at macroscopic distances toward the symmetric point. Natural disorder usually does not exhibit the mirror symmetry. Nevertheless, the proposed mechanism of tunneling is not of pure academic interest, since it may be observed also in a classical system-a random electrical circuit where the symmetry can be artificially introduced. In what follows, we propose a method of secure communications based on the symmetry-induced mechanism of tunneling. The merit of this method is that it does not require a coding-decoding procedure.

To demonstrate the main idea of the symmetry-induced tunneling, we consider the tight-binding Anderson model ${ }^{4}$

$$
\psi_{n+1}+\psi_{n-1}=\left(E+\epsilon_{n}\right) \psi_{n},
$$

where $E$ is the eigenenergy and $\epsilon_{n}$ is the on-site energy.

Schrodinger Eq. (1) gives an exact description of the electrical circuit of classical impedances $Z_{n}$ and $z_{n}$ shown in Fig.
1. Application of Kirchhoff's loop rule to three successive unit cells of the circuit leads to the following linear relation between the currents circulating in the $(n-1)$ th, $n$ th, and ( $n$ $+1)$ th cells:

$$
z_{n} I_{n+1}+z_{n-1} I_{n-1}=\left(Z_{n}+z_{n}+z_{n+1}\right) I_{n} .
$$

If the vertical impedances are all the same, $z_{n}=z_{0}$, this equation is reduced to the tight-binding model with diagonal disorder [Eq. (1)] with $\epsilon_{n}=\delta_{n} / z_{0}$ and $E=2+Z_{0} / z_{0}$. Here the impedance $Z_{n}$ is split into mean value $Z_{0}=\left\langle Z_{n}\right\rangle$ and fluctuating part $\delta_{n}=Z_{n}-Z_{0}$.

This exact correspondence allows testing of quantum effects of Anderson localization using classical electrical circuits with random elements. In fact, during the last decade, chaotic resonant cavities have been successfully used for testing the predictions of quantum chaos. ${ }^{5}$ It is worth mentioning that electrical circuits have been widely used for modeling different physical phenomena. The first application of the method of equivalent circuits probably goes back to Lord Kelvin who used a discrete RC chain to study a signal transmission through a transatlantic cable. Many bright examples of electrical circuits that model quantum-mechanical behavior for simple but fundamental systems are given in the book by Pippard. ${ }^{6}$ Recently, it was proposed that the electromagnetic waveguide can be used to model such an exotic effect as Hawking black-hole radiation. ${ }^{7}$ Some effects of correlated disorder have been studied in the experiments with microwave propagation through disordered waveguides ${ }^{8}$ and subterahertz response of superconducting multilayers. ${ }^{9}$ Experimental realization of a system with desirable correlations and observation of the localized and extended states is much

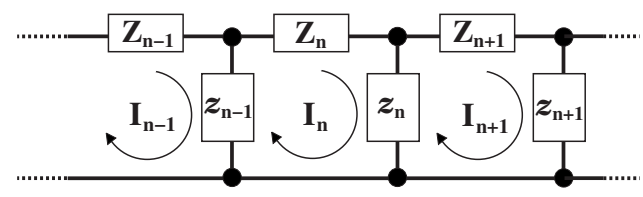

FIG. 1. Segment of infinite electric circuit of classical impedances. 


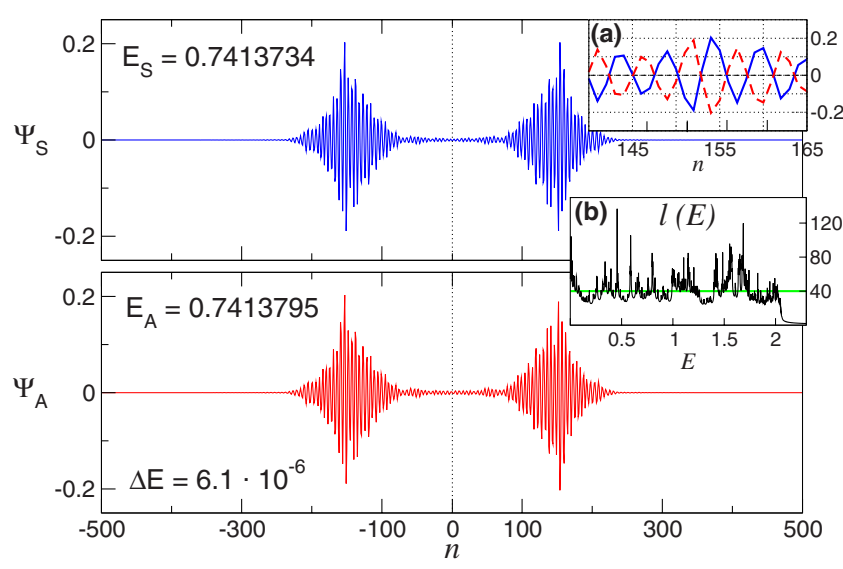

FIG. 2. (Color online) Two eigenstates with different parity $\left(\Psi_{S}\right.$ is even and $\Psi_{A}$ is odd) in random symmetric potential $\epsilon_{-n}=\epsilon_{n}$ with $\left\langle\epsilon_{n}\right\rangle=0$ and $\left\langle\epsilon_{n}^{2}\right\rangle=\epsilon_{0}^{2}=0.1$. These states belong to a doublet with energy splitting $\Delta E$. Insets: (a) Blow up of the right peaks of the eigenfunctions showing that they possess different parity. (b) $\mathrm{Nu}-$ merical result for the localization length as compared to the energyindependent function $l(E)=40$. The compensation of the energy dependence in $l_{0}(E)$ is not of principal importance and is done only to simplify the discussion of the numerical results.

easier in electromagnetic devices ${ }^{8,9}$ than in heterostructures with intentionally introduced disorder. ${ }^{10}$

\section{TIGHT-BINDING MODEL WITH SYMMETRIC POTENTIAL}

If the potential in Eq. (1) is an even function, $\epsilon_{n}=\epsilon_{-n}$, the eigenfunction $\Psi_{n}^{\alpha}$ is either an even or odd function of $n$. If an eigenfunction $\Psi_{n}^{\alpha}$ is localized near a site $n_{0}$, the amplitude of this state at the origin is exponentially suppressed, $\Psi_{n=0}^{\alpha}$ $\propto \exp \left[-\left|n_{0}\right| / l\left(E_{\alpha}\right)\right]$, provided $\left|n_{0}\right| \gg l\left(E_{\alpha}\right)$. However, due to definite parity of the wave function, another peak appears at the symmetric point $n=-n_{0}$. Strong localization of any excitation in the random potential is a result of destructive interference between propagating and backscattered waves. The appearance of the symmetric peak can be explained as a result of constructive interference. It leads to exponential increase of the amplitude of the wave, i.e., to antilocalization. ${ }^{11}$

In Fig. 2, we show two quasidegenerate eigenstates calculated for the symmetric potential of 1000 sites (i.e., only 500 of these sites are independent). The inverse localization length (the Lyapunov exponent) can be estimated from the formula ${ }^{3}$

$$
l^{-1}(E)=l_{0}^{-1}(E) \varphi(\mu), \quad \varphi(\mu)=1+2 \sum_{k=1}^{\infty} \xi(k) \cos (2 \mu k) .
$$

Here, $l_{0}^{-1}(E)=\epsilon_{0}^{2} /\left(8 \sin ^{2} \mu\right)$ is the Thouless ${ }^{12}$ result for the white-noise disorder, the function $\varphi(\mu)$ accounts for the contribution of correlations with correlation function $\left\langle\epsilon_{n} \epsilon_{n+k}\right\rangle$ $=\epsilon_{0}^{2} \xi(k)$, and the dispersion relation is $E=2 \cos \mu$. The results shown in Fig. 2 are obtained not for white noise but for slightly correlated disorder with correlator $\xi(1)=-1 / 2$ and $\xi(k>1)=0$. These short-range correlations are introduced in order to compensate for the smooth energy dependence of $l_{0}(E)$. It is easy to see that the contribution of the term with $k=1$ in Eq. (3) provides a flat dependence $l^{-1}(E)=\epsilon_{0}^{2} / 4$ $=$ const. Inset (b) in Fig. 2 shows the numerical values of $l(E)$, fluctuating around 40, in agreement with Eq. (3).

The energy spectrum of Eq. (1) with symmetric random potential is similar to the spectrum of a double-well potential. It consists of discrete levels, most of them lying within the interval $-2<E<2$. The energy levels are arranged in doublets of states with different parity. The energy $\delta(E)$ between the doublets scales with the length of the system $N$ as $1 / N$. The energy splitting $\Delta E$ in the doublet is exponentially small, $\Delta(E) \propto \exp \left[-2\left|n_{0}\right| / l(E)\right]$, i.e., the states are quasidegenerate. Both $\delta(E)$ and $\Delta(E)$ fluctuate with energy.

The symmetry-induced tunneling can be observed in the dynamics of an excitation. Let a perturbation be applied at one of the sites of the symmetric random sequence. In the simplest case, the perturbation is a $\delta$ excitation at the site $n_{0}$, $\psi_{n}(t=0)=\delta_{n n_{0}}$. Since this excitation is not an eigenfunction of the system, its temporal evolution is represented as a superposition,

$$
\psi_{n}(t)=\sum_{\alpha} C_{n_{0}}^{\alpha} \Psi_{n}^{\alpha} \exp \left(-i E_{\alpha} t\right)
$$

The sum in Eq. (4) runs over the eigenstates, which are all localized. The eigenstates that are centered closer to the initial excitation contribute more because the coefficient $C_{n_{0}}^{\alpha}$ $=\left\langle\Psi_{n}^{\alpha} \mid \psi_{n}(t=0)\right\rangle=\Psi_{n_{0}}^{\alpha}$ is the overlapping integral between the initial excitation and the eigenstate $\Psi_{n}^{\alpha}$. Let the eigenstates with maximum overlapping be $\Psi_{A}$ and $\Psi_{S}$. They form a doublet with the central energy $\bar{E}=\left(E_{A}+E_{S}\right) / 2$ and splitting $\Delta E=E_{A}-E_{S}$. Taking into account only these two terms in Eq. (4), the following approximate result for the evolution of the initial excitation can be easily obtained:

$$
\psi_{n}(t) \approx \frac{e^{-i \bar{E} t}}{\sqrt{l(\bar{E})}}\left[\cos \left(\frac{\Delta E}{2} t\right) \Psi_{+}(n)+i \sin \left(\frac{\Delta E}{2} t\right) \Psi_{-}(n)\right]
$$

Here, $\Psi_{ \pm}(n)=\left(\Psi_{S} \pm \Psi_{A}\right) / \sqrt{2}$. Each of these linear combinations is a single-peak function. The peak of $\Psi_{+}$is always close to the point of initial excitation. For the eigenfunctions shown in Fig. 2, the peak $\Psi_{+}$is localized in the region of negative $n$.

At the early stage of evolution, the initial $\delta$ peak at $n_{0}$ quickly spreads over the region of width $2 l(\bar{E}) \approx 80$ [see Fig. 3(a)]. Further spreading is suppressed by Anderson localization. Equation (5) becomes valid at a much later stage of the evolution when the essential part of the $\delta$ packet tunnels at the macroscopic distance $2\left|n_{0}\right|$ and the peak emerges at the symmetrical point $-\left|n_{0}\right|$. Two instants of this slow tunneling are shown in Figs. 3(b) and 3(c). The time of tunneling or the period of oscillations of the density $\left|\psi_{n}(t)\right|^{2}$ is $T=2 \pi / \Delta E$. If the distance $2\left|n_{0}\right|$ between the peaks exceeds the localization length, this time is large, $T \propto \exp \left[2\left|n_{0}\right| / l(E)\right]$ and the amplitude of the wave function at the origin is small 


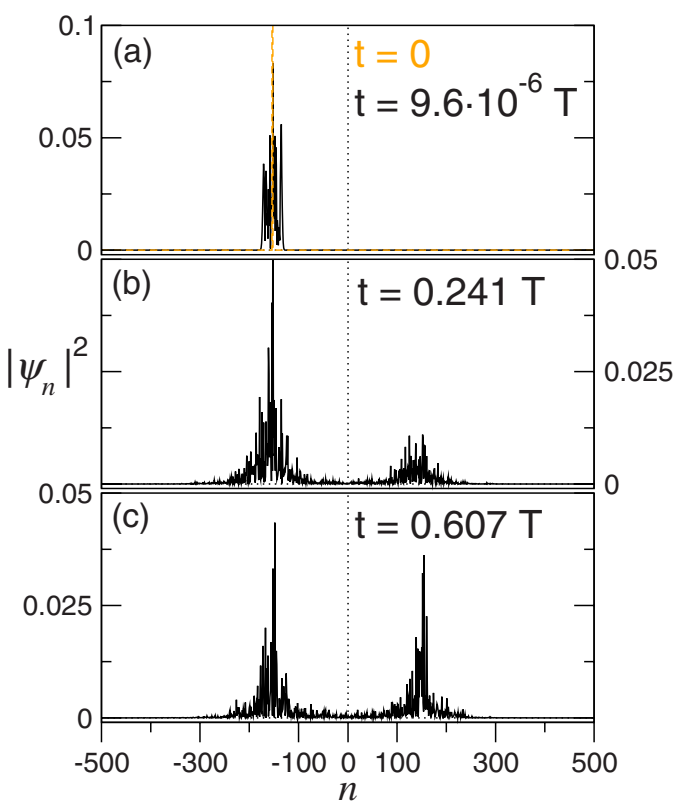

FIG. 3. (Color online) Spatial distribution of the probability $\left|\psi_{n}(t)\right|^{2}$ at three instants: (a) Spreading of the initial peak at the transient stage, $t=9.6 \times 10^{-6} \mathrm{~T}$. The initial peak of amplitude 1 at $n_{0}=-153$ is shown in gray. The secondary peak is not visible at this stage. (b) The secondary peak at $-n_{0}=153$ is well developed at $t$ $=0.241 \mathrm{~T}$. (c) The two peaks become almost equal at $t=0.607 \mathrm{~T}$.

$\propto \exp \left[-\left|n_{0}\right| / l(\bar{E})\right]$. The wave function, however, grows exponentially toward the symmetrical point $-n_{0}$. This increase is a manifestation of the tunneling induced by the symmetry. The dynamics of penetration of the initial excitation to the symmetrical point is very similar to the tunneling through a potential barrier, although there is no real barrier for weak disorder $\epsilon_{0}^{2} \ll 1$. Exponential behavior of the envelope of the wave functions is due to multiple-scattering events. One can speak about an effective double-well potential, which produces the same energy spectrum. Calculation of the parameters of this effective potential is a challenging inversescattering problem. The dynamics of tunneling through a real barrier with fluctuating parameters has been studied in Ref. 13. Tunneling processes without a real barrier are known in dynamical systems, where quantum transitions occur either between strongly localized states ${ }^{14}$ or between classically separated regions in phase space. ${ }^{15}$ It is worth mentioning that regular Bloch-type oscillations in a potential with correlated disorder may also occur due to the presence of two mobility edges in the energy spectrum. ${ }^{16}$

\section{SYMMETRIC RANDOM CIRCUIT}

Application of the proposed ideas to random electrical circuits is straightforward. In what follows, we demonstrate the evolution of the signal in a circuit shown in Fig. 1, with vertical impedances being equal to solenoids with $z_{n}$ $=-i \omega L_{0}$ and horizontal impedances being capacitors with fluctuating $Z_{n}=i /\left(\omega C_{n}\right) \approx\left(i / \omega C_{0}\right)\left(1-\delta C_{n} / C_{0}\right)$. Here, $C_{n}$ $=C_{0}+\delta C_{n}$ and $C_{n}=C_{-n}$. Propagation of an excitation in this circuit follows the equation

$$
C_{n+1} \ddot{V}_{n+1}+C_{n-1} \ddot{V}_{n-1}-2 C_{n} \ddot{V}_{n}=V_{n} / L_{0},
$$

where $V_{n}$ is the voltage drop at the $n$th capacitor. This equation requires two initial conditions. Let the voltage drop $V_{0}$ be applied at $t=0$ to the capacitor $C_{n_{0}}$, inducing the initial current $I_{0}=C_{n_{0}} \dot{V}_{0}$. Stationary solutions $\left[V_{n} \propto \exp (-i \omega t)\right]$ are either even or odd functions and the spectrum of eigenfrequencies consists of a set of doublets. For an infinite chain, the majority of the eigenfrequencies occupy an interval $\left[\omega_{0} / 2, \infty\right]$, where the resonant frequency $\omega_{0}=\left(L_{0} C_{0}\right)^{-1 / 2}$. Assuming that the initial perturbation excites only the closest to the site $n_{0}$ pair of eigenstates $\left(V_{A}\right.$ and $\left.V_{S}\right)$, the solution of Eq. (6) can be written in a form similar to Eq. (5),

$$
\begin{aligned}
V_{n}(t) \approx & \sqrt{\frac{1}{C_{0} l(\bar{\omega})}}\left\{\left[C_{n_{0}} V_{0} \sin (\bar{\omega} t)\right.\right. \\
& \left.-\frac{I_{0}}{\bar{\omega}} \cos (\bar{\omega} t)\right] \sin \left(\frac{\Delta \omega}{2} t\right) V_{-}(n)+\left[C_{n_{0}} V_{0} \cos (\bar{\omega} t)\right. \\
& \left.\left.+\frac{I_{0}}{\bar{\omega}} \sin (\bar{\omega} t)\right] \cos \left(\frac{\Delta \omega}{2} t\right) V_{+}(n)\right\} .
\end{aligned}
$$

Here, $\bar{\omega}$ is the center of the doublet and $\Delta \omega$ is the frequency splitting. Single-peak functions $V_{ \pm}(n)=\left(V_{S} \pm V_{A}\right) / \sqrt{2}$ play the same role as $\Psi_{ \pm}$do in Eq. (5). Equation (7) shows that the evolution of the initial signal in a random (symmetric) electrical circuit is similar to the wave-packet evolution obtained from the tight-binding model. There is an obvious symmetry-induced tunneling of the initial signal at macroscopic distances.

The effect of tunneling can be used for secure communications. Instead of coding and decoding a signal, we propose to suppress the transmitted signal by a filter containing a circuit with random elements. The filter is inserted between the emitter output and the transmitting line. The signal, suppressed to the level of noise, can be safely transmitted to a receiver over a transmitting line of arbitrary length. At the input of the receiver, the signal is restored using another filter, which is the symmetric counterpart of the output random circuit. The input filter restores only the signal (not noise), since constructive interference occurs only for the coherent part, which has passed through the output filter at the emitter. The noncoherent part (noise or any irrelevant signal) will be exponentially suppressed by the receiving random circuit. The two identical random circuits may be fabricated as microchips, which are installed (or replaced) simultaneously at the emitter and receiver. In the absence of dissipation and asymmetry between the two random elements, the proposed method guarantees a robust restoration of the signal. Inevitable Joule losses in the transmitting line have to be compensated for by an amplifier, which does not destroy the coherency of the signal.

A disadvantage of this method is the low rate of transmission. It is the prize one always pays for security. If the output signal is exponentially suppressed, the rate of transmission becomes exponentially low. Finite resistance of each resonant contour, which is neglected in the previous consideration, also limits the transmission rate. Nevertheless, our nu- 


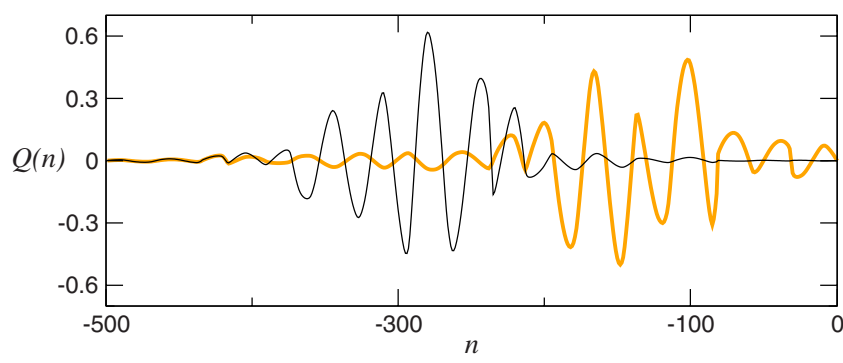

FIG. 4. (Color online) Distribution of charges (arbitrary units) for two doublets with $\bar{\omega} \approx 154 \mathrm{MHz}, T \approx 60 \mathrm{~ms}, 2\left|n_{0}\right|=470$ (thin line) and $\bar{\omega} \approx 162 \mathrm{MHz}, T \approx 39 \mu \mathrm{s}, 2\left|n_{0}\right|=162$ (thick line) in one of the possible realizations of a random symmetrical circuit containing 500 pairs of resonant contours. Only one eigenstate for each doublet is shown.

merical analysis shows that a reasonable compromise between low rate and high security is possible.

Let us consider a symmetric circuit of 500 pairs of resonant contours with $L_{0}=50 \mu \mathrm{H}, C_{0}=30 \mathrm{pF}$, and $\left\langle\delta C_{n}\right\rangle^{2}$ $=200(\mathrm{pF})^{2}$. Let the contours be fabricated of high quality elements and possess the $Q$ factor $5 \times 10^{3}$. The signal may tunnel through the random circuit if the tunneling time $T$ does not exceed the decay time $2 \pi Q / \omega_{0}$, where the resonant frequency $\omega_{0}=25 \mathrm{MHz}$. This gives the low limit for the splitting, $\Delta \omega / \omega_{0} \approx \exp \left(-2\left|n_{0}\right| / l\right)>1 / Q=2 \times 10^{-4}$. Now one obtains that the distance $2\left|n_{0}\right|$ between the centers of tunneling cannot exceed $l(\omega) \ln Q \approx 8.5 l(\omega)$. At the same time, $2\left|n_{0}\right|$ cannot be very short, otherwise the signal passes through the filter without sufficient suppression. Assuming that the power of the signal at the center of the symmetric circuit (which is the point where the output signal enters into the transmitting line) is reduced to not less than $e^{4} \approx 55$ times, the following interval for $2\left|n_{0}\right|$ is obtained: $4 l(\omega)<2\left|n_{0}\right|$ $<8.5 l(\omega)$. For the random circuit with aforementioned parameters, we found at least five doublets that satisfy this inequality. They are centered at different sites and have different localization lengths that lead to different tunneling times. In Fig. 4, we show the distributions of charges $Q(n)$ for two doublets with tunneling times of $39 \mu$ s and $60 \mathrm{~ms}$. Since the overlapping between these eigenstates is weak, they may provide different levels of security and rates of transmission. Moreover, each realization of a finite set of random values of $\delta C_{n}$, taken from the same statistical ensemble, gives rise to different regimes. Thus, the appropriate regime can always be found.

In our analysis, we ignored the decoherence effects, which are inevitable at finite temperatures. In order for the electron tunneling between the symmetric points to be observable, the distance $2\left|n_{0}\right|$ between them cannot be less than the localization length $l(E)$ and, at the same time, cannot exceed the phase-breaking length $l_{\phi}$,

$$
l(E) \ll 2\left|n_{0}\right| \ll l_{\phi} .
$$

In truly one-dimensional systems, where only forward- and backward-scattering processes are allowed, the localization length is always of the order of the elastic mean free path, $l(E) \sim l_{e}$. Real estimates of the scattering mechanisms in natural one-dimensional systems (e.g., molecular wires) show that $l_{e} \sim l_{\phi}$ at $T=1-2 \mathrm{~K} .{ }^{17}$ This means that Eq. (8) can be hardly satisfied, at least at a few kelvin. However, in artificial superlattices, such as those fabricated in Refs. 9 and 10 , the localization length $l(E)$ can be strongly reduced due to correlated disorder. ${ }^{18}$ This mechanism of enhancement of localization length may help in producing states with very small $l(E)$, thus giving a possibility for the condition (8) to be realized in superlattices.

There are two more effects, temperature smearing ${ }^{19}$ and hopping conductivity, ${ }^{20}$ that may destroy the localized regime of electron transport. Fortunately, this is not the case for photons with wavelength in the infrared region and longer. There is also no decoherence caused by temperature for electromagnetic waves, since its phase is not affected by lattice fluctuations. The only essential result of temperature on the propagation of electromagnetic waves (or electrical signals) is damping due to Joule losses. This makes possible observation of many quantum effects at room temperatures. ${ }^{5,8,18}$

\section{CONCLUSIONS}

In conclusion, we demonstrate that in a symmetric random potential, the localized quantum states have two peaks as is required by the parity. Fast spreading of the initial $\delta$ excitation within localization length is followed by slow growth due to tunneling at the symmetrical point. This effect opens a possibility for secure communications that does not require coding and decoding of the transmitting signal. The random circuits may operate in a wide range of radio frequencies, using commercial capacitors and inductors. They can also be fabricated and operated in the near infrared region using the concept of plasmonic nanoelements proposed in Ref. 21 or random optical resonators. In the latter case, the $Q$ factor may be as large as $10^{10}$, i.e., dissipation does not impose a limitation for the level of security, allowing very strong suppression of the signal.

\section{ACKNOWLEDGMENTS}

This work is supported by the following grants: DOE Contract No. ER46312, CONACyT Contract No. 43730, MMA Contract No. A106/2007, MEC Contract No. FIS2006-00716, and JCyL Contract No. SA052A07. E.D. and A.R. acknowledge support from the Spanish Government under Ramon y Cajal and Jose Castillejo contracts (JC2007-00303). 
${ }^{1}$ J. C. Flores, J. Phys.: Condens. Matter 1, 8471 (1989); D. H. Dunlap, H.-L. Wu, and P. W. Phillips, Phys. Rev. Lett. 65, 88 (1990); P. Phillips and H.-L. Wu, Science 252, 1805 (1991); E. Lazo and M. E. Onell, Physica B 299, 173 (2001); W. Zhang and S. E. Ulloa, Phys. Rev. B 69, 153203 (2004); M. Titov and H. Schomerus, Phys. Rev. Lett. 95, 126602 (2005).

${ }^{2}$ J. M. Luck, Phys. Rev. B 39, 5834 (1989); F. A. B. F. de Moura and M. L. Lyra, Phys. Rev. Lett. 81, 3735 (1998); J. W. Kantelhardt, S. Russ, A. Bunde, S. Havlin, and I. Webman, ibid. 84, 198 (2000).

${ }^{3}$ F. M. Izrailev and A. A. Krokhin, Phys. Rev. Lett. 82, 4062 (1999); F. M. Izrailev, A. A. Krokhin, and S. E. Ulloa, Phys. Rev. B 63, 041102(R) (2001); F. M. Izrailev and N. M. Makarov, J. Phys. A 38, 10613 (2005).

${ }^{4}$ P. Anderson, Phys. Rev. 109, 1492 (1958).

${ }^{5}$ H.-J. Stöckmann, Quantum Chaos: An Introduction (Cambridge University Press, Cambridge, UK, 1999).

${ }^{6}$ A. B. Pippard, The Physics of Vibrations (Cambridge University Press, Cambridge, UK, 2007).

${ }^{7}$ R. Schützhold and W. G. Unruh, Phys. Rev. Lett. 95, 031301 (2005).

${ }^{8}$ U. Kuhl, F. M. Izrailev, A. A. Krokhin, and H.-J. Stöckmann, Appl. Phys. Lett. 77, 633 (2000).

${ }^{9}$ V. A. Yampol'skii, S. Savelev, O. V. Usatenko, S. S. Melnik, F. V. Kusmartsev, A. A. Krokhin, and F. Nori, Phys. Rev. B 75, 014527 (2007).

${ }^{10}$ V. Bellani, E. Diez, R. Hey, L. Toni, L. Tarricone, G. B. Parravi- cini, F. Domínguez-Adame, and R. Gómez-Alcalá, Phys. Rev. Lett. 82, 2159 (1999).

${ }^{11}$ The symmetric points are always correlated, $\left\langle\epsilon_{n} \epsilon_{-n}\right\rangle=\epsilon_{0}^{2}$. However, the nearest sites may be uncorrelated, giving rise to a nonstationary correlation function.

${ }^{12}$ D. J. Thouless, Phys. Rev. Lett. 61, 2141 (1988).

${ }^{13}$ V. Freilikher, M. Pustilnik, and I. Yurkevich, Phys. Rev. B 53, 7413 (1996).

${ }^{14}$ G. Casati, R. Graham, I. Guarneri, and F. Izrailev, Phys. Lett. A 190, 159 (1994).

${ }^{15}$ V. A. Podolskiy and E. E. Narimanov, Phys. Rev. Lett. 91, 263601 (2003)

${ }^{16}$ F. Domínguez-Adame, V. A. Malyshev, F. A. B. F. de Moura, and M. L. Lyra, Phys. Rev. Lett. 91, 197402 (2003); F. A. B. F. de Moura, M. L. Lyra, F. Domínguez-Adame, and V. A. Malyshev, Phys. Rev. B 71, 104303 (2005).

${ }^{17}$ Y. Imry, Introduction to Mesoscopic Physics (Oxford University Press, Oxford, UK, 1997).

${ }^{18}$ U. Kuhl, F. M. Izrailev, and A. A. Krokhin, Phys. Rev. Lett. 100, 126402 (2008).

${ }^{19}$ M. Moško, P. Vagner, M. Bajdich, and T. Schäpers, Phys. Rev. Lett. 91, 136803 (2003).

${ }^{20}$ M. E. Gershenson, Yu. B. Khavin, A. G. Mikhalchuk, H. M. Bozler, and A. L. Bogdanov, Phys. Rev. Lett. 79, 725 (1997).

${ }^{21}$ N. Engheta, A. Salandrino, and A. Alú, Phys. Rev. Lett. 95, 095504 (2005). 